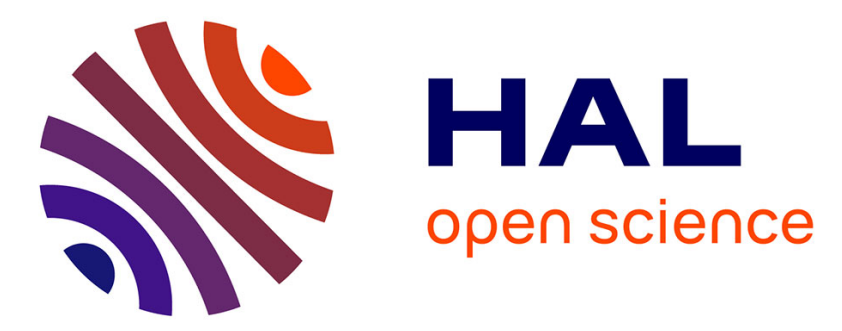

\title{
Very Sensitive Nanocalorimetry of Small Mass Systems and Glassy Materials
}

\author{
J.-L. Garden, A. Tavakoli, T. Nguyen-Duc, A. Frydman, Mohcine Laarraj, \\ Jacques Richard, Olivier Bourgeois
}

\section{- To cite this version:}

J.-L. Garden, A. Tavakoli, T. Nguyen-Duc, A. Frydman, Mohcine Laarraj, et al.. Very Sensitive Nanocalorimetry of Small Mass Systems and Glassy Materials. Janez Bonča, Sergei Kruchinin. Nanomaterials for Security, Springer, pp.35-44, 2016, 978-94-017-7591-5. 10.1007/978-94-017-7593-9_4 . hal-01359990

\section{HAL Id: hal-01359990 https://hal.science/hal-01359990}

Submitted on 5 Sep 2016

HAL is a multi-disciplinary open access archive for the deposit and dissemination of scientific research documents, whether they are published or not. The documents may come from teaching and research institutions in France or abroad, or from public or private research centers.
L'archive ouverte pluridisciplinaire HAL, est destinée au dépôt et à la diffusion de documents scientifiques de niveau recherche, publiés ou non, émanant des établissements d'enseignement et de recherche français ou étrangers, des laboratoires publics ou privés. 


\title{
Very sensitive nanocalorimetry of small mass systems and glassy materials
}

\author{
J.-L. Garden ${ }^{1,2}$, A. Tavakoli ${ }^{1,2}$, T. Nguyen-Duc ${ }^{1,2}$, A. Frydman ${ }^{1,2,3}$, M. \\ Laarraj $^{1,2}$, J. Richard ${ }^{1,2}$ and O. Bourgeois ${ }^{1,2}$ \\ 1 Institut NÉEL, CNRS, 25 avenue des Martyrs, F-38042 Grenoble France \\ 2 Univ. Grenoble Alpes, Inst NEEL, F-38042 Grenoble France \\ 3 Department of Physics, Bar-Ilan University, Ramat Gan 52900 Israel \\ jean-luc.garden@neel.cnrs.fr \\ olivier.bourgeois@neel.cnrs.fr
}

\begin{abstract}
Nanocalorimetry is a technique that deals with any thermal measurement methods in which either the samples to be studied have a size in the range of the nanometer scale or the measured energies involved are of the order of the nanojoule or below [1]. In this paper, we show the results of two nanocalorimetric experiments. The first one is related to the measurement of specific heat on ultra-thin small systems (thin films) at low temperature. It is shown that such measurement can be sensitive to less than one monolayer of materials. The second one illustrates the efficiency of calorimetric studies sensitive at the nanoJoule on complex system (polymeric glass) at room temperature. We then discuss the potentiality of these experimental methods in the field of security: the measurement of either a very small mass or very small quantity of energy for the detection of tiny thermal events.
\end{abstract}

\subsection{Introduction}

The calorimetric techniques are the experimental methods that allows the measurement of heat exchanges during a phase transition or any change in matter (temperature, pressure, volume etc...). It is a versatile method used for materials characterization that spans a wide range of domains going from low temperature physics to room temperature biology [1]. Part of the calorimetric techniques are dedicated to the measurement of the specific heat, one of the most important thermal properties of materials along with the thermal conductivity. The specific heat of a system reveals its intrinsic property by quantifying the amount of heat necessary to increase the temperature of the system by one Kelvin. Hence, nanocalorimetry is the part of calorimetry allowing the measurement of very small systems (nanometer scale) or very small quantities of heat (nanoJoule range). As an illustration, we will present in this article two sensitive nanocalorimetric measurements of the specific heat of very different systems: the first concerns the measurement of the specific 
heat of very thin superconducting layers (few mono-atomic layers). The second is about the measurement of very fine specific heat variation during the relaxation of a glassy polymeric system. These two examples of nanocalorimetric measurement will enlighten its general interest in the thermal detection of small systems or small thermal events.

\subsection{Nanocalorimetric measurement of nanometric thick layers}

The purpose of this first part is to illustrate how sensitive specific heat measurements can help detecting a very small mass change. By using ac calorimetry, we can indeed detect the heat capacity of materials in Joule per Kelvin, linearly dependent on the mass of the sample. In this experiment, based on a silicon membrane sensor [2], we demonstrate that the measurement of monoatomic layer of materials is possible. Necessarily, measuring the specific heat of very thin film is a major experimental challenge. Indeed, it involves the measurement of very small mass samples requiring a very high sensitivity, here at low temperature. This will be made possible thanks to recent experimental developments of highly sensitive specific heat measurement equipment [3-5].

\subsubsection{Experimental set-up}

A new apparatus has been made to measure the heat capacity of quench condensed thin films. Quench condensed films are obtained by thermally evaporating materials in-situ directly on the thermal sensor [5-8] at low temperature. This is allowing the deposition of a fraction of monolayer of materials in order to test the actual sensitivity of the calorimetric cell; This has been done here for superconducting materials but has been also performed for magnetic one [9]. The system has been tested by evaporating pure $\mathrm{Pb}(99.99 \%)$ on the membrane that is regulated at $8 \mathrm{~K}$. The fabrication of the calorimetric sensor has been described elsewhere, we will only highlight its very peculiarities $[2,3,5]$. The sensitive part is composed of a thin silicon membrane ( $5 \mu \mathrm{m}$ thick) on which a $\mathrm{NbN}$ thermometer [10] and a $\mathrm{Cu}$ heater have been lithographied to allow thermal measurements (see Fig. 1.1). The total heat capacity measurement that we will show below represents the heat capacity of the membrane itself along with the thermometer and heater on which the heat capacity of the sample is added. We keep the absolute values measured for the sake of simplicity.

\subsubsection{Results and discussions}

In this experiment, we have done sequential evaporations and measure the heat capacity for every of them (see Fig. 1.2). The size of the sample is $3 \mathrm{~mm}$ 


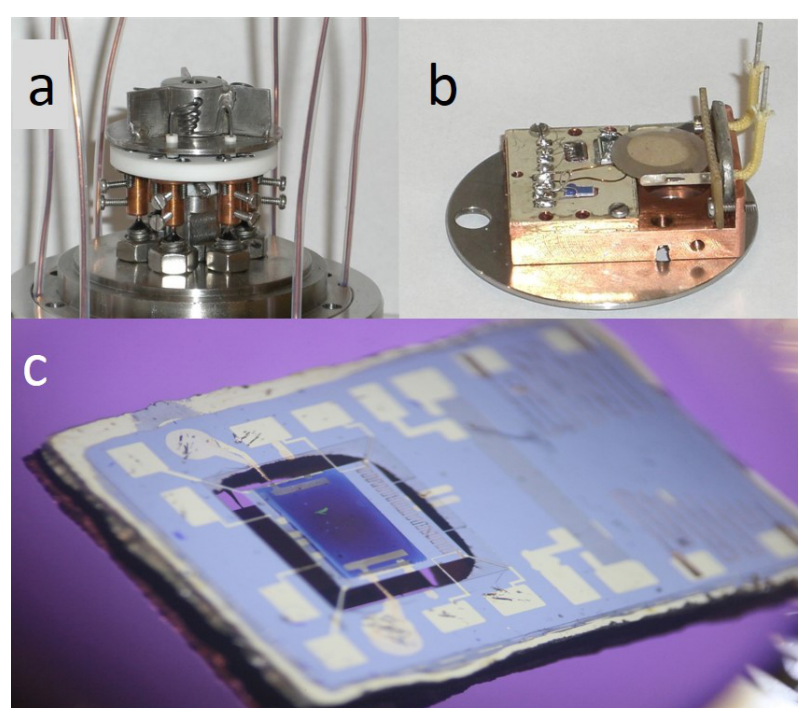

Fig. 1.1. a. Picture of the bottom part of the evaporation chamber. The baskets used for the quench condensation of $\mathrm{Pb}$ materials can be seen. $\mathrm{b}$. Picture of the sample holder part were the silicon membrane is located on the back of the regulation stage and the quartz crystal used for the measurement of mass sample. c. A photograph of a silicon membrane after an evaporation located between the heater and the thermometer of the membrane. The electrodes used for the electrical characterization of the evaporated samples are seen on the two sides of the membrane

by $2 \mathrm{~mm}$, and hence since a monolayer (ML) of $\mathrm{Pb}$ represents $0.18 \mathrm{~nm}$, one ML corresponds approximately to 10 nanogram. Each thermal evaporation of metal can be easily distinguished from the previous one from the $C_{p}$ data. As expected, the heat capacity increases as the thickness (and the number of ML) of the thin films increases. It is important to stress here that only that experiment allows the measurement of ultra-thin layer thanks to the cold evaporation of materials. Any evaporation at room temperature would severely damage the quality of the sample rendering any data interpretation very difficult.

The table gathers the different experimental values that can be extracted from the measurement at $5.55 \mathrm{~K}$ for the different evaporation of $\mathrm{Pb}$. The successive materials depositions (between the 5th and the 8th) show clearly the quality of the measurement. Having a sensitivity of the order of $\pm 0.01 \mathrm{~nJ} / \mathrm{K}$, it is clear that a film thickness smaller than one ML can be detected. To illustrate more clearly that point, we have calculated in the table the corresponding number of monolayers measured as a function of the sequential depositions. It appears that this experimental set-up is able to detect a variation of mass of less than one ML; basically on the order of 0.5ML. This 


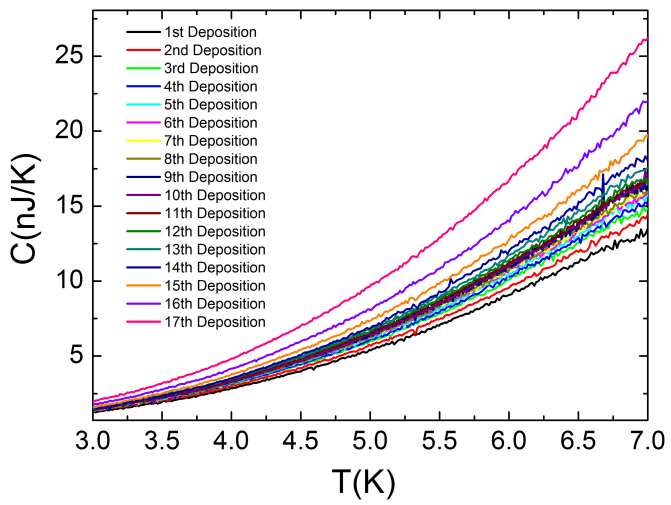

Fig. 1.2. Heat capacity measurement versus temperature for various successive evaporation of $\mathrm{Pb}$ on the silicon membrane. The heat capacity is continuously increasing as materials is added. Each evaporation from the 3rd to next correspond approximately to less than one ML

number is definitely beyond the state of the art in terms of highly sensitive specific heat measurement at low temperature.

Similar developments have been done using silicon nitride membrane as the mechanical support for samples instead of silicon. We have also recently demonstrated very sensitive measurement using this new sensor since mass of less than 1 nanogram can be detected.

\subsection{3 conclusions}

We have developed an innovative equipment that allows the measurement of heat capacity of cold evaporated thin films that is sensitive to sub-monolayer change of materials thickness. It can be used for the study of $\mathrm{Bi}, \mathrm{Sn}, \mathrm{In}, \mathrm{Pb}$ or

Table 1.1. This table gathers the different experimental values that can be extracted from the measurement at $5.55 \mathrm{~K}$. The first column gives the number of the evaporation, the second the overall heat capacity (including the contribution of the sensor), the third column the thickness of the film, the fourth the number of ML, the fifth the mass (in microgram) and the last column shows the sensitivity in $\mathrm{J} / \mathrm{K}$

\begin{tabular}{llllll}
\hline Evap. $\sharp$ & $C_{p}(\mathrm{~nJ} / \mathrm{K})$ & $\mathrm{t}(\mathrm{nm})$ & $\mathrm{ML}$ & $\mathrm{m}(\mu \mathrm{g})$ & $\Delta C_{p}$ \\
\hline 5 th & 8.37 & 10.3 & 57.2 & 0.48 & \pm 0.01 \\
6th & 8.50 & 10.46 & 58.1 & 0.487 & \pm 0.01 \\
7th & 8.54 & 10.51 & 58.4 & 0.49 & \pm 0.01 \\
8th & 8.62 & 10.61 & 58.9 & 0.494 & \pm 0.01 \\
\hline
\end{tabular}


any other materials that can be quench-condensed at low temperature. This experiment show the possibility of performing the $C_{p}$ measurements successively by increasing the thickness of the films in-situ. Very or ultra-thin films can be measured using this experiment especially for the study of heat capacity signature of phase transition in very low dimensional systems. It is also a clear demonstration that ultra-small mass variation can be detected from a calorimetric tool that has been optimized; this is paving the way for new detection schemes based on highly sensitive thermal measurements.

\subsection{Nanocalorimetric measurement of glassy polymer}

The glass transition is the transformation of a liquid that has been supercooled (by high cooling rates for example) into a disordered solid called a glass. From a fundamental point of view, this transformation is still opened to questions particularly because glassy systems are outside thermodynamic equilibrium. In this case, some thermodynamic state variables of the glass depend on time. For example, the enthalpy or entropy of a glass decreases along time whether the system is maintained at constant pressure and temperature. Calorimetry is a usual method to study the glass transition. In particular, during the glass transition (vitrification) the heat capacity of the system undergoes a jump. During successive heating, the jump is recovered along with an enthalpy peak which reflects the decrease in energy that has occurred during the previous cooling. This quantity is analogous to the energy furnished to the system in order to recover the initial state (liquid state). Classical calorimetry fails to give new insights on this phenomenon partly because its lack of resolution. Here we present the results of nanocalorimetric experiments on a classical polymeric glass-former, the poly(vinyl acetate) PVAc. The main result reside in the recording of very fine decreases of the heat capacity during aging at a constant temperature below the glass transition temperature, $\mathrm{Tg}$.

\subsubsection{The nanocalorimeter}

The nanocalorimeter has been described in the reference [11]. It is composed of three different parts: -the first one is constituted by a microsensor made by means of microfabrication technologies (see Fig. 1.3). It is composed of two half-cells, one containing a thermometer and the other containing a heater. The sample is taken as a sandwich between these two half-cells ensuring good thermal contacts between sample, thermometer and heater. An oscillating thermal power is generated into the sample by the heater, and the resulting temperature oscillation is recorded with the thermometer at the same frequency. -the second one is an home-made low noise and highly stable electronic detection chain allowing oscillating power generation along with oscillating temperature detection at the same frequency with very high sensitivity. 


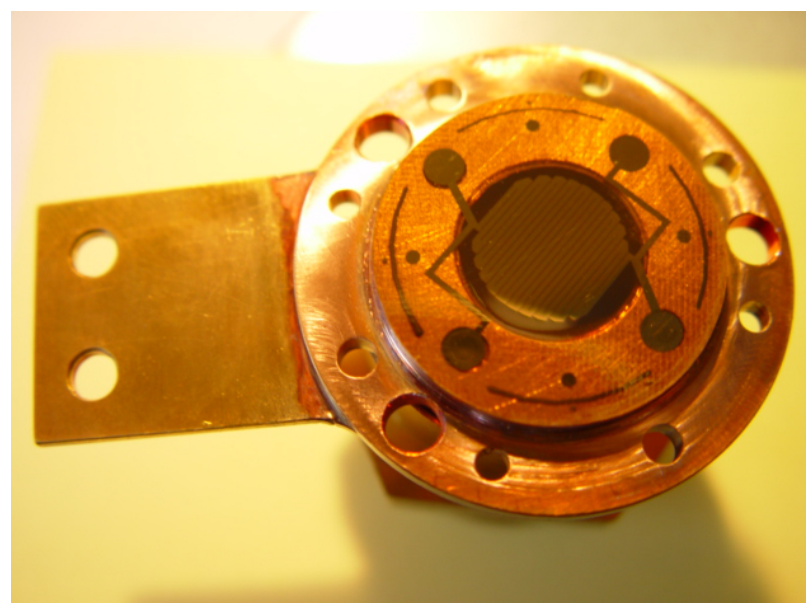

Fig. 1.3. Half-cell of the nanocalorimeter with patterned thin film heater in coppernickel on a 25 micrometer thick polyimide membrane stuck on a copper piece

-the third one is an home-made thermal surroundings dedicated to the sensor and the electronic chain. It is composed of different thermally regulated shields under vacuum inside an external stage in stainless steel.

The nanocalorimetric method of detection is the so-called ac-calorimetry [12] which consisting in the recording of a temperature oscillation (amplitude and phase) with the thermometer after supplying an ac-power in the cell by means of a heater (see Fig. 1.3). The temperature oscillation is recorded versus time and numerical fast Fourier transform (FFT) provides the amplitude and phase. The measurement is carried out following different experimental protocols (cooling, heating, aging,...) having different thermal histories. In particular, the amplitude of the sample temperature oscillations gives rise to the modulus of a complex heat capacity by means of the equation:

$$
\left|C_{p}^{*}\right|=\frac{P_{0}}{\omega\left|T_{a c}\right|} .
$$

\subsubsection{Results}

In Fig. 1.4, we present the modulus of the complex heat capacity and the phase of the temperature oscillation recorded with the nanocalorimeter during a heating $\operatorname{ramp}(d T / d t=+0.2 \mathrm{~K} / \mathrm{min})$ of the glassy polymeric sample (PVAc) undergoing a glass transition. The modulus of the complex heat capacity shows a typical jump during heating due to the unfreezing of the molecular movements when the glass is transforming into a liquid. The phase exhibits a anomaly with a peak superimposed to the jump. The high resolution of the measurement is illustrated by the quality of the baselines either in the glassy or in the liquid state for the modulus and the phase. 

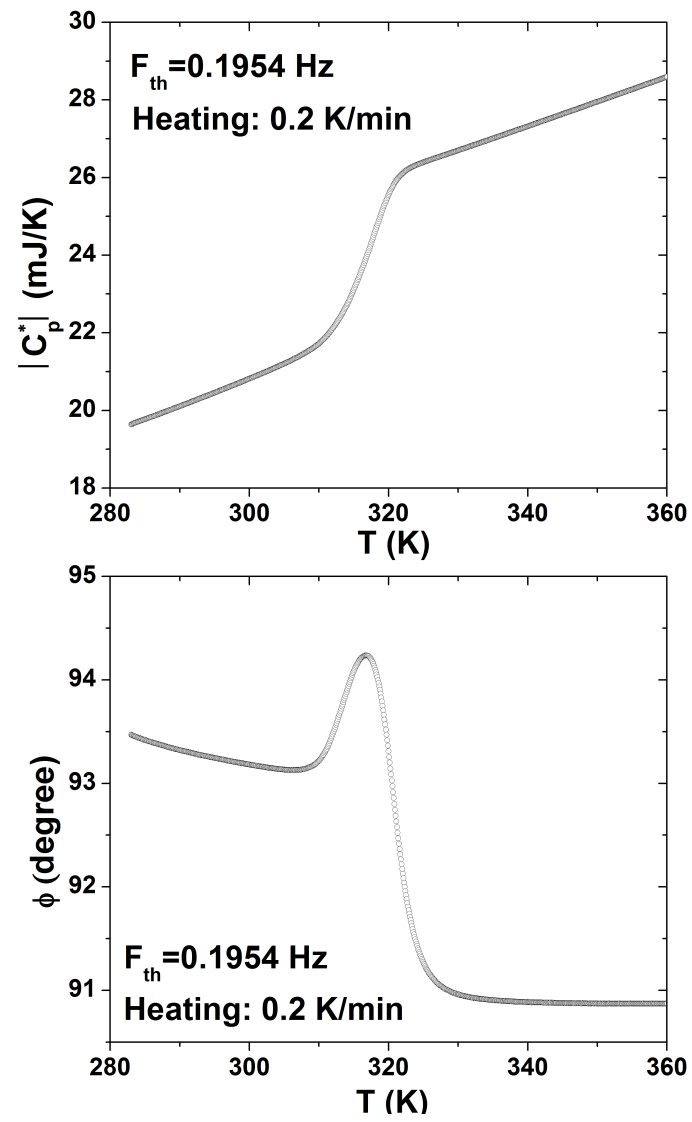

Fig. 1.4. The modulus of the complex heat capacity and the phase of the temperature oscillation are represented as a function of temperature during a heating ramp of $+0.2 \mathrm{~K} / \mathrm{min}$ between $283 \mathrm{~K}$ to $360 \mathrm{~K}$. The frequency of the temperature oscillation is of $0.1954 \mathrm{~Hz}$ with an amplitude of the order of $450 \mathrm{mK}$. The sample has been previously cooled between these two temperatures at a rate of $-1.2 \mathrm{~K} / \mathrm{min}$.

In Fig. 1.5, the resolution in percent is directly shown on the modulus of the complex heat capacity when the measurement is carried out during hundred minutes at a constant temperature of $339 \mathrm{~K}$. The resolution is the noise (peak to peak) divided by the absolute value of the modulus of the complex heat capacity for each experimental point $\Delta\left|C_{P}^{*}\right| /\left|C_{P}^{*}\right|$. The graph is directly given in percent showing that the nanocalorimeter is about one thousand times better than classical temperature oscillating calorimeters having a resolution of about $1 \%$. The minimum heat capacity variation during a thermal event that can be detected with the calorimeter is above $500 \mathrm{~nJ} / \mathrm{K}$. 


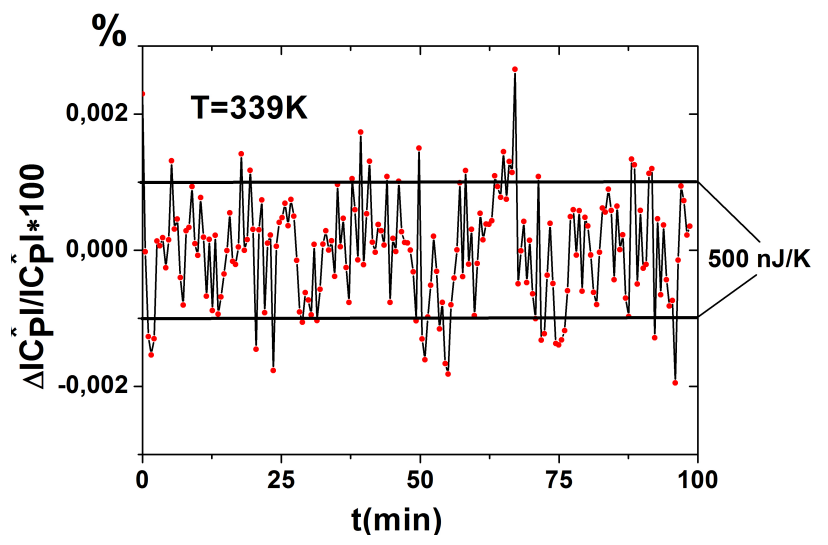

Fig. 1.5. Resolution in percent on the modulus of the complex heat capacity when the temperature oscillations are recorded during hundred minutes at a constant temperature of $339 \mathrm{~K}$.

In Fig. 1.6, we present the slow decrease of the modulus of the complex heat capacity along time when the PVAc sample is maintained at a constant temperature of $297 \mathrm{~K}$ during more than three hours. The graph is presented using a logarithmic scale showing that the decrease slows down progressively but does not stop. This decrease is a consequence of the aging process (decrease of the glass energy) when the temperature is maintained constant below the glass transition temperature. This is typical of a glassy system. The point that we want to stress here is the significant variation of $C_{p}$ during such a time interval. The relative variation is of the order of $1.2 \%$ on the aging time. That is to say that this decrease cannot be measured with classical calorimeters having a resolution of few percents. It is important to mention that, over the same time window, no temperature drift of the sample has been recorder. As a conclusion, these two last examples highlight the capability of the nanocalorimeter of detecting very fine heat capacity variations during the evolution in time of slowly relaxing complex systems.

\subsection{Discussion}

In this paper, we have presented two different experiments in the fields of nanocalorimetry. The first one deals with measurement of very thin layers at low temperature while the second one deals with measurement of complex systems at room temperature. These typical examples show that it is possible to reach the nanojoule per kelvin range with high sensitive calorimetry. By definition, calorimetry measure any kind of energy variation with temperature of any materials or any phase transformation of materials (specific 


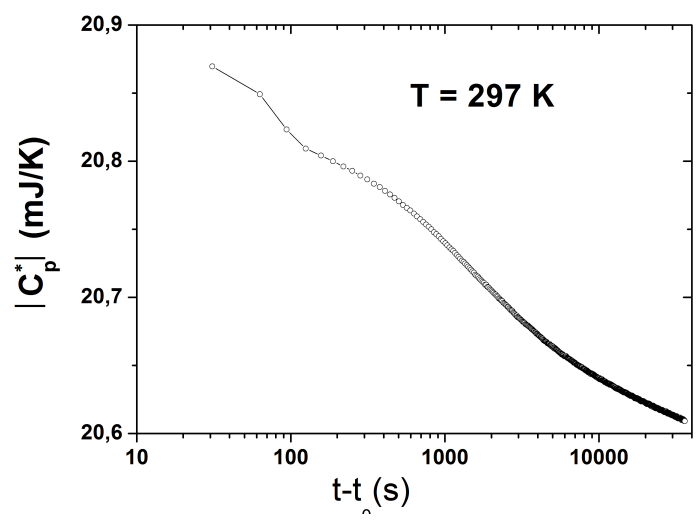

Fig. 1.6. Modulus of the complex heat capacity along time in logarithm scale during a aging experiment at the constant temperature of $297 \mathrm{~K}$

heat, enthalpy, entropy changes etc...). We thus can imagine that such highly sensitive method of detection can find applications in the fields of security by its capability of measuring down-scaled nanosystems. We can however list different topics where nanocalorimetry can help in the detection process:

- detection of fine molecular interactions in liquids (pharmacy, drug detection, contamination of water, doping substances detection,...)

- detection of small amount of toxic gas (gas sensor, ...)

- detection of small quantities (particles, gas) of explosives

Acknowledgments We acknowledge technical supports from Nanofab, the Cryogenic and the Electronic facilities and the Pole Capteur Thermométrique et Calorimétrie of Institut Néel for these experiments. Funding for this project was provided by LANEF, Laboratoire d'Excellence, for the senior grant of Aviad Frydman, and by the CNRS (Mission pour l'interdisciplinarité) by means of the call DEFI Instrumentation aux Limites.

\section{References}

1. J.-L. Garden, H. Guillou, A.F. Lopeandia, J. Richard, J.-S. Heron, G.M. Souche, F.R. Ong, B.Vianay, O. Bourgeois, Thermochim. Acta 492, 1628 (2009).

2. F. Fominaya, T. Fournier, P. Gandit, and J. Chaussy, Rev. Sci. Instrum. 68, 4191 (1997).

3. O. Bourgeois, S.E. Skipetrov, F. Ong and J. Chaussy, Phys. Rev. Lett. 94, 057007 (2005).

4. F.R. Ong, O. Bourgeois, Europhys. Lett. 79, 67003 (2007). 
5. S. Poran, M. Molina-Ruiz, A. Gerardin, A. Frydman, and O. Bourgeois, Rev. Sci. Instrum. 85, 053903 (2014).

6. J.P. Garno, Rev. Sci. Instrum. 49, 1218 (1978).

7. O. Bourgeois, and R. C. Dynes, Phys. Rev. B 65, 144503 (2002).

8. O. Bourgeois, A. Frydman and R.C Dynes, Phys. Rev. Lett. 88, 186403 (2002).

9. M. Molina Ruiz, A.F. Lopeandia, F. Pi, D. Givord, O. Bourgeois, and J. Rodriguez-Viejo, Phys. Rev. B 83, 140407(R) (2011).

10. O. Bourgeois, E. André, C. Macovei, J. Chaussy, Rev. Sci. Instrum. 77, 126108 (2006).

11. M. Laarraj, R. Adhiri, S. Ouaskit, M. Moussetad, C. Guttin, J. Richard, and J.-L. Garden, Rev. Sci. Instrum. 86, 115110 (2015).

12. P. Sullivan and G. Seidel, Phys. Rev. 173, 679 (1968) 